\title{
Prosthetic posterior teeth with cusps may improve patient satisfaction with complete dentures
}

\author{
Do different occlusal schemes affect patient satisfaction in subjects who require \\ complete dentures?
}

\begin{abstract}
Sutton AF, Glenny AM, McCord JF. Interventions for replacing missing teeth: denture chewing surface designs in edentulous people. Cochrane Database of Systematic Reviews 2005, issue 1 Art. No.: CD004941.pub2.
\end{abstract}

Data sources The Cochrane Oral Health Group Trials Register, Cochrane Central Register of Controlled Trials, Medline, OLDMedline, Embase, Zetoc (Zetoc provides access to the British Library's electronic table of contents of approx. 20,000 current journals and 16,000 conference proceedings published per year. The database covers 1993 to date and is updated daily. It includes an e-mail alert service to enable you to keep up-to-date with relevant new articles and papers), System for Information on Grey Literature in Europe (SIGLE) (SIGLE is a bibliographic database covering European non-conventional (or 'grey') literature in the fields of pure and applied science and technology, economics, social sciences and humanities), and the Science Citation Index were used to source studies. Reference lists of identified, relevant trials and review articles were scanned. Unpublished data were sought through personal contact with experts in the field. There was no language restriction.

Study selection Randomised controlled trials (RCT) or quasi-RCT were selected that recruited edentulous adults and compared complete dentures with different occlusal schemes, with regard to patient satisfaction and masticatory function.

Data extraction and synthesis The quality assessment of included trials was undertaken independently and in duplicate by two reviewers. Data were also extracted by two reviewers independently. Disagreements were discussed and a third reviewer consulted as necessary. Authors were contacted for clarification or missing information. Data were excluded until further clarification if agreement could not be reached.

Results Thirteen trials were thought to be potentially relevant. Ten of these studies were subsequently excluded following further analysis. Two trials require further information from the author before being considered eligible for inclusion. Only one crossover trial $(n=30)$, which compared lingualised teeth and zero-degree teeth, fully met the review's inclusion criteria. Twenty patients preferred the lingualised denture, five the zero-degree denture and five patients had no preference. There was a statistically significant difference in favour of the lingualised denture with an odds ratio of 10.00 (95\% confidence interval, 2.04-48.96).

Conclusions There is weak evidence that it may be advantageous for dentists who provide a complete denture service to prescribe prosthetic posterior teeth with cusps, rather than cuspless teeth, to improve patient satisfaction. This conclusion may only be made tentatively until further, well-conducted trials are undertaken that compare different occlusal schemes for complete dentures.

Address for correspondence: Emma Tavender, Review Group Co-ordinator, Cochrane Oral Health Group, Manchester Dental Education Centre, School of Dentistry, University of Manchester, Higher Cambridge Street, Manchester M15 6FH, UK. E-mail: emma.tavender@man.ac.uk

\section{Commentary}

Stable and retentive complete dentures enhance patient satisfaction. The appropriate design of the various surfaces contributes to stability and retention by resisting displacing forces. During mastication, swallowing and parafunction, displacing forces on dentures become unfavourable when they overwhelm their intrinsic retention and stability causing discomfort, trauma and, hence, poor functional and aesthetic consequences. Factors enhancing denture-displacing forces therefore reduce patient satisfaction.

The form of the occlusal surfaces of denture teeth and the nature of their occlusal contacts are among the factors that significantly influence denture displacement. For this reason, since the first dentures were made, efforts have continued to find the most appropriate occlusal form and tooth arrangement. No conclusive evidence in this regard has ever been provided, however. Among the many reasons for this are the inappropriate approaches to investigations on the subject, drawing evidence from theoretical assumptions, use of subjective data, and inadequate scientific experimentation by not using acceptable controls. When, as a result, there is no evidence for a superior tooth form and arrangement, the practitioner is left at liberty to follow, and practise routinely, the least complicated approach. ${ }^{1}$

This systematic review aimed to find evidence for patient satisfaction, in terms of restored aesthetics, comfort and masticatory efficiency, with the use of a particular posterior denture tooth forms in complete dentures.

In this search for the available evidence on the topic, the reviewers have conducted an extensive exploration and critical reading of the literature. A common problem was that many studies focused on chewing efficiency rather than patient satisfaction as the main outcome. Determination of masticatory efficiency with complete dentures is only one of the several parameters of patient satisfaction and better masticatory efficiency with a particular tooth form and arrangement may not necessarily reflect overall satisfaction of a patient using dentures.

Bearing this in mind, along with the well-chosen inclusion and exclusion criteria, only 13 out of the 1076 titles identified were thought potentially relevant. Only one ${ }^{2}$ could eventually be included. This is a point worth noting when evaluating the strength of the evidence for the observed superiority of the anatomic and cusped posterior denture teeth in terms of patients satisfaction: that evidence is the findings of a single study, the quality of which the reviewers have very rightly criticised.

The key points to emerge form this review are a confirmation of the lack of and the need for well-conducted RCT in restorative dentistry, and the authors' recommendations for future work in this area. Despite identifying a lack of evidence this review can be seen as preparing the groundwork for work that is nearing completion by one of the review authors (AF Sutton, unpublished data). Hopefully this review will help initiate RCT for, and bring scientific vigour to, the muchneeded assessment and understanding of outcomes of restorative interventions. 


\section{Practice point}

- Despite weak evidence of improved patient satisfaction with prosthetic posterior cusped teeth, dentists are advised to continue providing patients with complete dentures with cusped posterior teeth rather than using cuspless teeth.

\section{Fazal Ghani}

Department of Prosthodontics, Khyber College of Dentistry,

University Campus, Peshawar 25120, Pakistan
1. Lang BR. Complete denture occlusion. Dent Clin N Am 1996; 40:85-101.

2. Clough HE, Knodle JM, Leeper SH, Pudwill ML, Taylor DT. A comparison of lingualized occlusion and monoplane occlusion in complete dentures. J Prosthet Dent 1983; 50:176-179.

Evidence-Based Dentistry (2005) 6, 39-40.

doi:10.1038/sj.ebd.6400324 